\title{
eJRIEPS
}

Ejournal de la recherche sur l'intervention en éducation physique et sport

$24 \mid 2011$

Varia

\section{Chronogenèse des objets enseignés en basket-ball par quatre intervenants à l'école primaire}

Chronogenesis of basket-ball learning stuff by four teachers in primary school

\section{Odile Devos-Prieur}

\section{(2) OpenEdition}

\section{Journals}

Édition électronique

URL : http://journals.openedition.org/ejrieps/4064

DOI : 10.4000/ejrieps.4064

ISSN : 2105-0821

Éditeur

ELLIADD

Référence électronique

Odile Devos-Prieur, «Chronogenèse des objets enseignés en basket-ball par quatre intervenants à l'école primaire », eJRIEPS [En ligne], 24 | 2011, mis en ligne le 01 juillet 2011, consulté le 10 décembre 2020. URL : http://journals.openedition.org/ejrieps/4064 ; DOI : https://doi.org/10.4000/ejrieps.4064

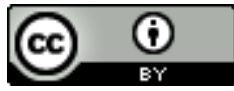

La revue eJRIEPS est mise à disposition selon les termes de la Creative Commons Attribution 4.0 International License. 


\section{eJRIEPS 24 juillet 2011}

Chronogenèse des objets enseignés en basket-ball par quatre intervenants à l'école primaire

Odile Devos-Prieur

IUFM Midi-Pyrénées, Université de Toulouse 2, UMR EFTS

\section{Résumé}

La recherche présentée s'intéresse à la chronogenèse des objets enseignés au fil du cycle de quatre professeurs d'école de formation différente: un maître basketteur, un maître "tout venant », un maître ayant une formation STAPS, un maître travaillant en partenariat avec un intervenant extérieur. L'hypothèse initiale est que les rapports aux savoirs des intervenants (Chevallard, 1989), leur formation initiale comme leur côtoiement de la pratique sociale du basket-ball, influencent la façon de concevoir les contenus d'enseignement. Les résultats mettent en évidence que les assujettissements des professeurs à leurs institutions de formation sont déterminants. Le maître STAPS et le maître tout venant sont portés par une intention dévoluante liée à leurs rapports à la formation et à l'enseignement. Le maître basketteur mobilise en acte des savoirs incorporés qui semblent faire défaut aux autres enseignants. Quant à l'intervenant extérieur, il est façonné par ses institutions de référence, le club de basket départemental et une formation ancienne et techniciste dans cette APS. La recherche met ainsi en évidence une grande variabilité des contenus enseignés selon les sites.

Mots-clés : Didactique, Rapports aux savoirs, Action du professeur, Education physique et Sportive, Basket-ball, Ecole primaire.

\section{Introduction}

Dans cet article, nous nous attachons à caractériser les liens entre les rapports aux savoirs de quatre intervenants (de parcours professionnels et personnels contrastés) et le développement des contenus d'enseignement au fil d'un cycle de basket-ball (BB) à l'école élémentaire. En effet, Baillat et Guillon (1998) pointent que dans 23\% des cas l'Education Physique et Sportive (EPS) est confiée à un intervenant extérieur issu du monde sportif, et que les enseignants peuvent formaliser les contenus de façon très 
eJRIEPS 24 juillet 2011

hétérogène dans cette discipline. Nous proposons donc de mener une analyse comparative du cycle d'enseignement conduit respectivement par :

- un enseignant qui a une expérience vécue en BB à un bon niveau,

- un enseignant qui a une licence Sciences et Techniques des Activités Physiques et Sportives (STAPS),

- un enseignant « tout venant »,

- un enseignant qui travaille avec un intervenant extérieur spécialiste BB.

En étudiant les manières dont ces quatre intervenants conçoivent et structurent leurs cycles de BB, nous souhaitons accéder à une partie de ce qu'Amade-Escot (1998), Amade-Escot et Marsenach (1995) appellent les «déterminations du système didactique », qui rendent compte dans son fonctionnement de phénomènes qui lui sont propres. Nous nous intéressons plus particulièrement aux déterminations qui s'exercent sur l'organisation temporelle d'un cycle d'enseignement. Nous examinons en quoi les rapports aux savoirs à enseigner des professeurs (ici relativement au BB) influencent le type d'objets mis à l'étude, leurs organisations et structurations au fil du cycle c'est-à-dire à la chronogenèse (Chevallard, 1991).

\section{Cadre général de la recherche : l'approche didactique de l'action du professeur}

S'intéresser à la chronogenèse au sens de Chevallard (1991) c'est-à-dire, rappelons le, à la succession des savoirs en BB au fil du cycle dans quatre systèmes didactiques contrastés impose d'identifier, à partir de l'action du professeur, quels sont les contenus réellement enseignés. A cet effet, nous décrivons l'action du maître, pendant toute la durée du cycle, en effectuant une analyse ascendante de la transposition didactique selon Schubauer-Léoni (2006) et Schubauer-Léoni \& Lettenegger (2002) qui stipulent que les contenus mis à l'étude sont à rechercher dans les dispositifs de formation proposés et dans l'activité effective du professeur et des élèves. Nous considérons que cette focale d'analyse permet à la fois de rendre compte et de révéler les rapports aux savoirs des intervenants, leurs incidences sur l'épistémologie pratique (Amade-Escot, Amans-Passaga \& Montaud, 2009; Sensevy, 2007), et en conséquence sur le processus d'enseignementapprentissage. II s'agit de mettre au jour ce qui dépend, selon Chevallard (1991), essentiellement de leur «topos », la conception des dispositifs mis à l'étude et leurs articulations sur le temps du cycle. Chevallard considère que tout sujet construit un « rapport institutionnel » spécifique à l'institution dans laquelle il agit. Mais chaque individu 


\section{eJRIEPS 24 juillet 2011}

appartient à différentes institutions et c'est de la confrontation de ces multiples rapports aux savoirs institutionnels qu'émerge la personne (Chevallard 1989). Dans cette étude, nous considérons que la diversité des « rapports institutionnels » (ici en BB) que les quatre intervenants entretiennent avec l'éducation physique s'actualisent sous forme d'un «rapport personnel» aux savoirs. En suivant Chevallard, nous considérons que le «rapport personnel» aux savoirs se façonne dans cette diversité de «rapports institutionnels». Les intervenants qui ont en charge l'enseignement de l'éducation physique à l'école primaire (professeur ou intervenant extérieur) ont ainsi construit un « rapport personnel » au BB par le biais des institutions traversées : l'éducation physique, le club, les loisirs, la famille, etc., comme l'a montré par ailleurs Brière-Guenoun (2005). Ce rapport personnel au BB va devoir s'actualiser dans le cadre institutionnel de l'éducation physique qu'ils doivent enseigner, et en conséquence va devoir vivre sous la contrainte du « contrat institutionnel » en usage dans le système didactique scolaire. Nous faisons donc l'hypothèse que le rapport personnel des intervenants vis-à-vis du BB à enseigner, même s'il est modifié par l'intermédiaire du contrat institutionnel en usage en éducation physique à l'école primaire, reste toujours lié aux expériences antérieures. C'est à partir de cette focale d'analyse, des rapports aux savoirs, que nous observerons la manière propre à chaque intervenant de programmer les objets enseignés au fil du cycle et de faire avancer le temps didactique à travers les dispositifs proposés au fil du cycle (Chevallard, 1991). Nous faisons l'hypothèse que les multiples rapports aux savoirs des intervenants les conduisent à structurer leur cycle de façon singulière, à programmer divers objets de savoir selon différents moments, à jouer sur la relation entre ancien et nouvel objet de savoir (Chevallard, 1999).

\section{Méthodologie}

Pour mener l'analyse des quatre cycles d'enseignement en éducation physique, nous avons opté pour une observation ethnographique sur toutes les séances qui les composent afin d'accéder à la diachronie du système didactique lors de leçons ordinaires. Selon Leutenegger (2000), une leçon ordinaire est une leçon qui appartient en propre au système didactique en «milieu naturel». Ce temps assez long rend possible l'identification de la chronogenèse mise en œuvre par les quatre intervenants.

Nous nous appuyons sur les principes méthodologiques de Schubauer-Leoni et Leutenegger (2002). Notre étude est clinique dans la mesure où nous nous proposons d'étudier la singularité des quatre systèmes didactiques sans intervenir pour modifier le 


\section{eJRIEPS 24 juillet 2011}

travail du maître et les situations qu'il propose. La composante expérimentale est légère et elle consiste à proposer un thème d'étude commun aux quatre maîtres que nous avons négocié avec eux lors d'un entretien préalable au cycle, dit «ante cycle ». En effet, il est important, selon Amade-Escot et Sensevy (2002), dès les premiers moments de la prise de contact, d'établir un contrat de recherche avec les acteurs de la recherche qui soit le plus explicite. Aussi, nous avons demandé aux quatre maîtres, de traiter un même thème d'étude dans le cycle afin de pouvoir comparer les objets de savoir réellement enseignés de chaque dispositif proposé, par les quatre sites au fil cycle : conserver la balle pour aller tirer en élargissant l'espace de jeu c'est-à-dire étirer « la grappe » entre les deux paniers.

3. 1. Le contexte de l'étude

Nous avons observé quatre classes de cours moyens (élèves de 9 à 10 ans) pendant un cycle de BB portant sur le thème d'étude qui traversera le cycle d'enseignement apprentissage (cf. supra), thème d'étude classique du cycle trois que les quatre intervenants ont accepté de mettre à l'étude, sous leur seule responsabilité, selon des modalités de travail qui leur appartiennent.

\section{2. Le choix des enseignants}

Nous avons retenu quatre profils d'intervenants contrastés, traduisant la pluralité des contextes aujourd'hui rencontrés à l'école élémentaire, et ayant une expérience dans l'enseignement. Ils ont tous plus de dix ans de pratique, ce qui a permis d'atténuer les problèmes du fonctionnement et de la gestion de la classe qui sont propres aux professeurs débutants (Durand, 1998). En revanche ces quatre intervenants ont un parcours de formation professionnel ou universitaire, et une expérience vécue dans l'activité physique enseignée qui sont différents. Au final, les participants à notre étude ont été les suivants : un maître ayant une expérience vécue d'un bon niveau en BB (sélection régionale Midi-Pyrénées en cadet et en junior), un maître titulaire d'une licence STAPS, un maître «tout-venant », enseignant et enfin un maître travaillant en partenariat avec un intervenant extérieur, titulaire d'un brevet fédéral en BB. Notre recherche consiste donc en une analyse de cas croisés, nous pensons toutefois qu'elle rend compte de possibles traits génériques des maîtres basketteurs, des maîtres «tout-venant », des maîtres STAPS, des intervenants extérieurs. Aussi, dans notre rédaction des résultats, pour dépersonnaliser l'étude et pour un respect de l'anonymat, nous emploierons ces dénominations: maître basketteur $[\mathrm{MB}]$, maître «tout-venant » [MTV], maître STAPS [MS], et maître et intervenant extérieur [M/IE].

3. 3. Recueil des données 


\section{eJRIEPS 24 juillet 2011}

Afin de décrire et de comprendre l'action didactique des enseignants, quatre types de données ont été recueillis. Tout d'abord, les notes au vol qui constituent le corpus central des données traitées dans cet article. Pour affiner certaines des interprétations, nous avons exploité les planifications et documents donnés aux élèves, les entretiens avec les intervenants à différents moments du cycle ainsi que les vidéos des séances. Ces trois derniers types de données ont permis de réduire les incertitudes des interprétations liées au carnet de bord.

\section{1. 1. Les notes au vol : carnet de bord}

Nous avons observé l'ensemble des quatre cycles et tenu un carnet de bord dans lequel nous notions les actions de l'enseignant: les dispositifs proposés aux élèves, les différentes consignes et variables de commande permettant aux élèves d'entrer dans les situations. Nous utiliserons les données qui sont susceptibles d'expliquer les choix transpositifs des enseignants, celles qui renseigneront sur les éléments chronogénétiques.

3. 1. 2. Les planifications des maîtres et fiches d'observation des élèves

Les planifications et les fiches d'observation des élèves, quand elles existaient, ont été des outils importants pour confirmer les objets enseignés des dispositifs proposés dans les séances et dans le cycle.

\section{1. 3. Les vidéos des séances}

Les trois vidéos des séances (début, milieu et fin de cycle) ont permis de revenir de façon plus précise sur certains ajustements. Nous avons notamment noté la manière dont les intervenants conçoivent l'articulation des situations entre elles et comment ils effectuent leurs régulations. Ces données ont permis d'affiner certaines de nos interprétations faites à partir des notes au vol sur la totalité du cycle.

\section{4. Les entretiens}

Nous avons retenu des entretiens ante et post (qui ont eu lieu lors des trois séances filmées) les éléments qui permettent de compléter le point de vue extrinsèque du chercheur en prenant en compte le point de vue intrinsèque des intervenants observés. Ainsi avons-nous pris en compte leurs choix d'objets d'enseignement et leurs choix de situations pour la durée du cycle. Ce dernier corpus a conforté nos interprétations.

\section{5. Traitement des données}

Nous avons réduit la grande quantité de données recueillies aux données pertinentes au regard de la problématique c'est-à-dire l'organisation des savoirs sur l'axe du temps didactique et la progressivité didactique mise en place par les enseignants en élaborant 


\section{eJRIEPS 24 juillet 2011}

" une matrice de condensation des données " (Huberman et Miles, 1991) sous-tendue par trois grands thèmes :

- La situation de référence proposée en début de cycle, son influence sur les objets enseignés pendant le cycle, l'avancée du temps didactique (la chronogenèse) et la façon dont elle est réactivée dans le cycle,

- La succession des objets enseignés et des situations qui les mettent en scène pendant le cycle,

- Les ruptures chronogénétiques c'est-à-dire relever les ruptures entre les objets enseignés au cours du cycle pendant le cycle s'il y en a.

Nous avons reconstruit la chronogenèse des cycles à partir de ces matrices de condensation des données. Dans la suite du texte, les segments de phrases entre / / renvoient à la dénomination faite par le chercheur des objets de savoir mis à l'étude. Ceux qui sont énoncés par les intervenants selon des formulations diverses seront présentés en italique. Nous souhaitons ainsi différencier le point de vue extrinsèque du chercheur du point de vue de l'intervenant observé dans la présentation des résultats.

\section{Résultats}

Pour rendre compte de la chronogenèse des quatre sites, nous avons élaboré un mode de condensation des données permettant d'avoir une vue synoptique du cycle. Chaque objet d'enseignement mis à l'étude est représenté par une couleur sur l'axe des ordonnées, et nous en déclinons les différents objets de savoir mis à l'étude tout au long du cycle. La connexité progressive des éléments mis à l'étude sur la durée du cycle est soulignée par une continuité des pavés de couleur, leurs ruptures didactiques par des traits pointillés verticaux. Les mises en situation de référence (match) sont indiquées en vert en dessous de la ligne du temps didactique (axe des abscisses). Parfois, elles sont accompagnées de chiffres entre parenthèses (6) correspondant au nombre de matches joués pendant la séance. Pour faciliter la lecture des tableaux synthétisant la chronogenèse des savoirs dans les quatre cycles, il convient de se référer à la légende suivante : 


\begin{tabular}{|l|l|}
\hline Sigles & Significations \\
\hline JPB : & Joueur Porteur de Balle \\
\hline JDB : & Joueur Destinataire de Balle \\
\hline JNPB : & Joueur Non Porteur de Balle \\
\hline D : & Défenseur \\
\hline CR : & Critère de réussite \\
\hline TT : & Tirs Tentés \\
\hline TR : & Tirs Réussis \\
\hline PB : & Possession de Balle \\
\hline S : & Séance \\
\hline RO : & Rebond offensif \\
\hline
\end{tabular}

La première séance du cycle correspondant à une évaluation diagnostique ne figure pas dans le tableau synoptique, elle sera néanmoins analysée dans cet article.

4. 1. Analyse de la chronogenèse des objets enseignés du maitre basketteur (MB) Tableau I. Matrice de condensation des données de la chronogenèse des objets de savoir enseignés par MB.

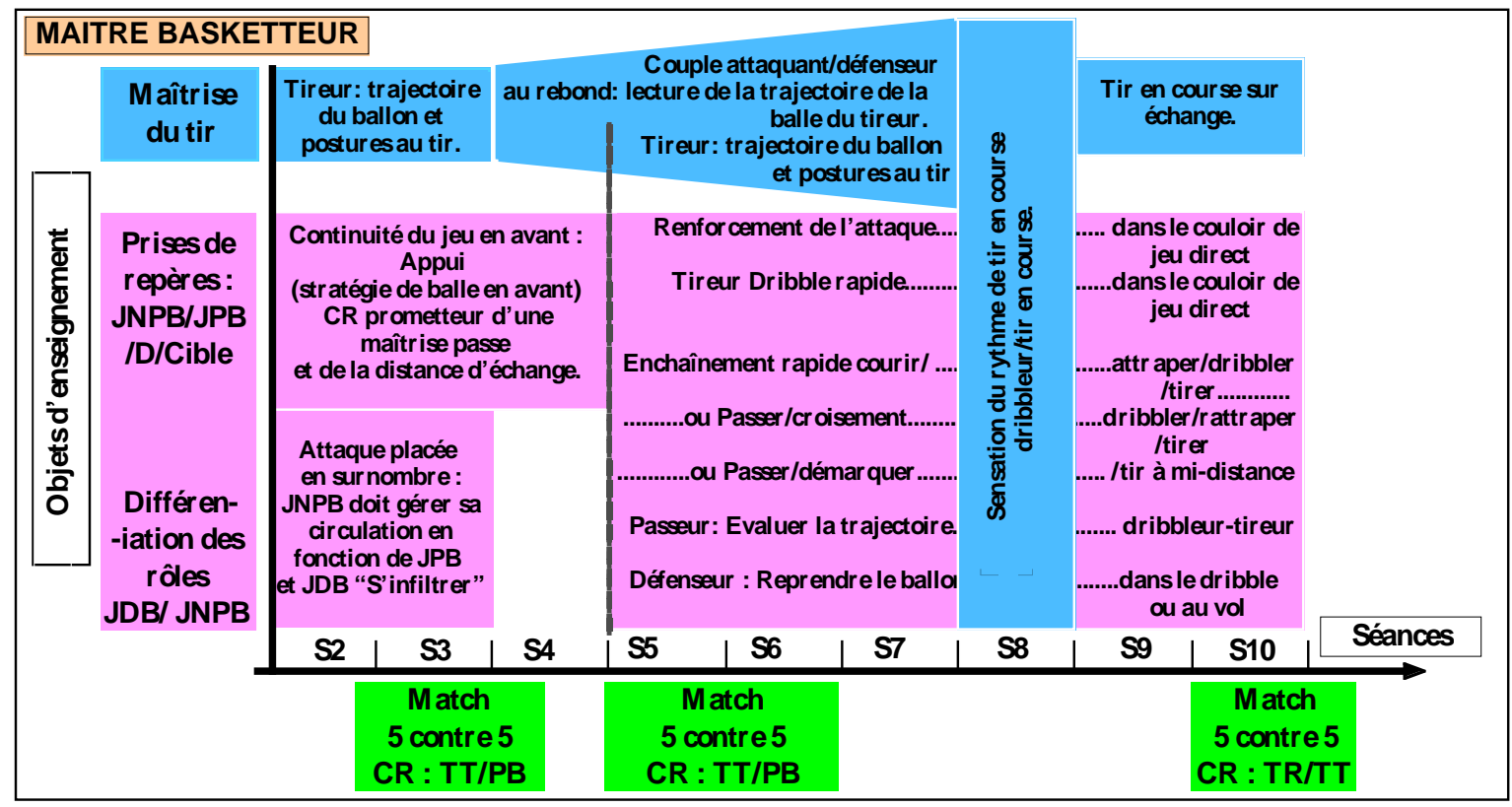

Notons que, dans ce tableau synthétique du MB les pointillés de par et d'autre de la colonne bleue : «..... » signifie la nécessité de continuer la lecture de l'objet savoir audelà de la colonne bleue de la séance 8. 


\section{eJRIEPS 24 juillet 2011}

Ce tableau synthétique met en évidence que deux enjeux de savoir sont mis à l'étude pendant tout le cycle du MB. Tout d'abord, l'analyse didactique met en évidence des objets enseignés et des situations qui correspondent au thème /Conserver la balle pour aller tirer en élargissant l'espace de jeu/ (en rose). Les analyses a priori montrent qu'ils sont essentiels dans ce thème. Ensuite, nous avons relevé des objets enseignés qui correspondent au thème /Etre efficace au tir et développer des positions intéressantes/ (en bleu). Les données disponibles montrent que ces deux thèmes sont articulés dans la situation de référence organisée par MB afin d'effectuer l'évaluation diagnostique. Chevallard (1992) indique que, en fonction des topos des élèves, de l'enseignant et de l'objet de savoir, le concept de milieu est institutionnellement formaté en fonction de la façon dont l'enseignant découpe son cycle de BB. C'est pourquoi le milieu, à cette échelle macroscopique d'analyse, est révélateur des gestes de l'enseignant qui sont contraints et déterminés par le rapport institutionnel à l'objet de savoir spécifique du BB. Nous considérons donc que ces deux thèmes sont chargés de sens pour les élèves car ils prennent en compte le gain du jeu (marquer un panier), et leur niveau de jeu (en termes de circulation des attaquants dans un rapport de force avec des défenseurs). Examinons maintenant la dynamique évolutive des savoirs mis à l'étude selon les différents moments du cycle.

4. 1. 1. Le premier moment du cycle : mise en scène introductive

La première séance a pour visée d'instaurer une première rencontre avec le problème que MB souhaite faire expliquer par ses élèves, c'est-à-dire la question stratégique de la circulation de la balle et des joueurs pour créer les conditions d'un tir tenté à chaque possession de balle. Le dispositif est présenté avec une contrainte qui amène les élèves à mettre en relation la stratégie utilisée par l'équipe et le degré de réussite au match. Le gain du match est en effet obtenu lorsque le nombre de tirs tentés sur le nombre de possessions de balle d'une équipe est supérieur à celui de l'équipe adverse. Ce critère de réussite permet aux équipes d'avoir accès au chemin qui leur reste à parcourir pour progresser. Ce type de tâches sera d'ailleurs repris tout au long du cycle.

4. 1. 2. Le deuxième moment du cycle : de la séance 2 à la séance 4

De la séance 2 à la séance 4, MB commence son cycle par l'exploration d'un type de tâches attaché à des enjeux de savoir relatifs aux stratégies collectives [dans deux situations à supériorité numérique (en rose) : le joueur relais (situation réversible) et 2 contre 1 (situation consistant en des traversées de terrain)], et un autre type de tâche ayant des enjeux de savoir relatifs aux habiletés individuelles (en bleu). Notons une 


\section{eJRIEPS 24 juillet 2011}

rupture didactique liée à ce dernier type de tâches qui sont décontextualisées en S2 et S3 et seront re-contextualisées à partir de S4 ("le Tireur Rebondeurs" (en bleu) : tir en appui avec duel sous le panier des deux rebondeurs, offensif et défensif pour la récupération du TT). Nonobstant, les objets enseignés de ces deux types de tâches (rose et bleu) sont articulés. En effet, la /Construction du point haut de la trajectoire/ de la passe (dans le joueur relais) est connexe avec celle du tireur (dans le tireur rebondeurs), et l'objet /Développer des positions favorables/ (les deux rebondeurs) est présent dans les deux situations.

4. 1. 3. Le troisième moment du cycle : de la séance 5 à la séance 10

De la séance 5 à la séance 10, MB introduit l'efficacité au tir dans le type de tâches attaché à l'enjeu de savoir relatif à /Prendre en compte « la forme » du couple attaquantdéfenseur pour prendre l'avantage / (dans la situation du retard du défenseur : pavé rose) qui est connexe avec l'objet mis à l'étude lors de ce moment didactique dans les situations du tireur rebondeurs de S5 à S7, et du tir en course (colonne bleue). Celui-ci occupe toute la séance 8 ce qui fait l'objet d'une deuxième rupture du cycle, de type chronogénétique. En effet de manière assumée, MB introduit un changement dans le critère de réussite : il s'agit maintenant de compter le nombre de TR sur le nombre de TT pendant les matches (en vert) et non plus comme dans les séances précédentes les TT sur le nombre de possessions de balle. Cette rupture accompagne l'introduction judicieuse de tir en course par rapport à l'avancée du savoir au fil du cycle.

4. 1. 4. Le moment de l'exploitation des savoirs mis à l'étude dans le jeu MB construit le bilan des deux moments précédents. II correspond à « des essais et des retouches» lors de la mise en œuvre des situations pendant ces deux moments didactiques: reprise de la situation à supériorité numérique tout au long du cycle (en rose), où seules les stratégies collectives efficaces sont conservées et valorisées dans les échanges avec les élèves, les autres stratégies étant progressivement oubliées. L'avancée didactique entre ces différents moments (moments de la technique et exploitation de la stratégie efficace) ne s'opère pas sans rupture (voir supra les ruptures décrites en 4.1.2 et 4.1.3).

\section{1. 5. Le moment de l'évaluation}

Pendant les séances 10 et 11, le moment de l'évaluation MB réussit à combiner les attentes différentielles entre élèves en maintenant les deux critères de réussite en situation de jeu (TT/PB et TR/TT). Rappelons qu'en séance 8 seul le critère de réussite TR/TT est pris en compte dans les situations de jeu (cf. la rupture chronogénétique de la 


\section{eJRIEPS 24 juillet 2011}

séance 8 (bleu) consacrée au tir en course) alors, que dans l'évaluation il donne la possibilité aux équipes les moins fortes de comptabiliser quelques points.

4. 1. 6. Conclusion sur la chronogenèse dans le cycle du maître basketteur Ce qui caractérise le cycle du MB, c'est le faible nombre d'objets de savoir mis à l'étude (2) qui sont articulés entre eux au sein de la séance dans la mesure où ils peuvent réutiliser les savoirs développés dans un atelier dans un autre atelier. Les séances sont aussi articulées entre-elles c'est-à-dire que les élèves peuvent utiliser les savoirs mis en jeu dans les ateliers d'une séance dans les séances qui la suivent.

4. 2. Analyse de la chronogenèse des objets enseignés du maître tout venant (MTV) Tableau II. Matrice de condensation des données de la chronogenèse des objets de savoir enseignés de MTV.

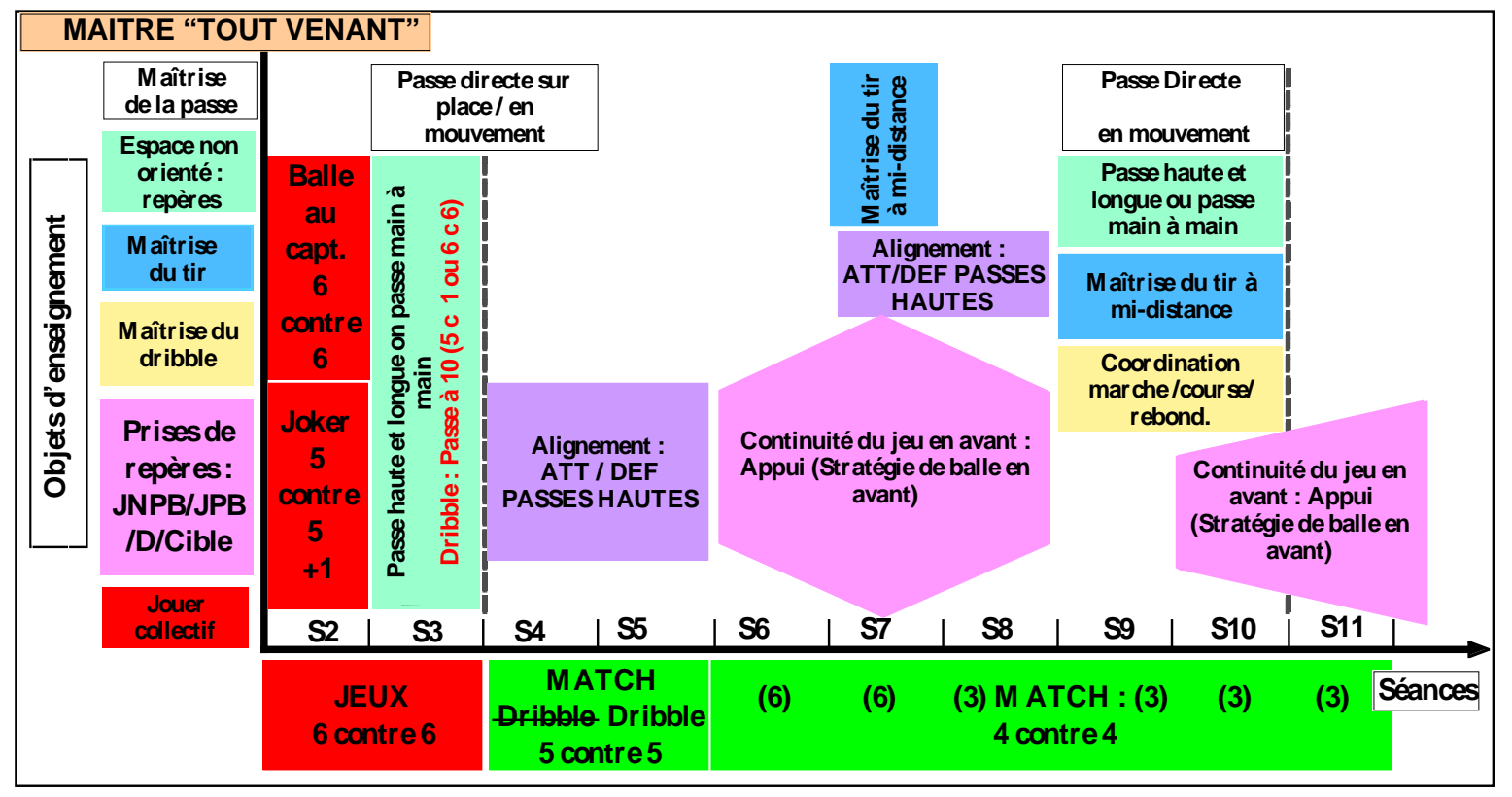

La condensation des données met en évidence une multiplicité des objets de savoir mis à l'étude tout au long du cycle avec une structuration assez chaotique. Ils sont introduits, à l'instar du MB, par une situation de référence et une première fiche d'observation (mettre en relation la stratégie utilisée par l'équipe et le degré de réussite au match en comptabilisant le nombre de TT sur le nombre de possession de balle) en relation avec le thème d'étude, /Conserver la balle pour tirer en élargissant l'espace de jeu/ (en rose). Ce thème occupe une place centrale dans le cycle du MTV. Toutefois, en analysant les situations didactiques qui mettent en scène les différents objets d'enseignement relatifs de ce thème, nous avons observé une discontinuité des séances qui les abritent, de la séance 4 à la séance 8 et de la séance 10 à la séance 11 (en rose plus ou moins foncé). 


\section{eJRIEPS 24 juillet 2011}

MTV introduit, pendant la deuxième séance, une nouvelle fiche d'observation: le sociogramme qui consiste en la distribution des échanges dans l'équipe. Cette fiche introduit l'étude du thème /Maîtrise des habiletés individuelles/ (en bleu, jaune et blanc) et plus particulièrement l'objet /Maîtrise de la passe/ (en blanc). C'est aussi la circulation entre le JPB et le JDB qui est étudiée à travers le sociogramme relativement au thème /Jouer collectif dans un espace qui n'est pas ou est orienté/ (en vert pâle) et à l'objet de savoir /Jouer collectif en /Augmentant la distance d'échange entre le JPB et le JDB/ (en rouge et en vert). II reste une des préoccupations du MTV pendant les onze séances du cycle.

Quelle est sa logique des objets enseignés tout au long du cycle? Selon quelle structuration?

4. 2. 1. Le recours à des jeux collectifs du début du cycle : un moment particulier de l'étude pour MTV

Les séances 2 et 3 sont une nouvelle première rencontre avec les thèmes d'enseignement du cycle (en rouge et vert pâle). Il s'agit de la balle au capitaine et de la passe à dix. Notre analyse pointe que ce premier moment est «un sous moment culturel » car, selon Chevallard (1999, p. 252), ces jeux sont « des mises en scènes de l'objet dans des "activités" qui en dépit de quelques traits culturels conservés [le terrain de BB, la passe, l'équipe...] n'ont qu'une relation assez relâchée », voire indépendante, avec le BB et sont par conséquent en rupture avec la suite des objets enseignés dans le cycle, il s'agit, selon nous, d'un compromis entre la conception d'apprentissage du MTV et le contrat de la recherche.

4. 2. 2 Le deuxième moment du cycle: une exploration des situations relatives aux différents objets enseignés pendant le cycle

Quatre objets, de la séance 4 à la séance 7, introduisent un approfondissement des techniques découvertes lors des séances précédentes : /Renforcement de la passe dans le couloir de jeu central/ (rose foncé), /Varier la distance de passe en fonction du rapport de force/ (rose clair) et les deux habiletés individuelles /Efficacité au tir/ (bleu) et /Efficacité à la passe/ (blanc). Les situations proposées posent des problèmes aux élèves car les configurations spatiales dans laquelle ils sont initialement placés [de type baby-foot (les trois défenseurs se déplacent, chacun sur des lignes étagées sur la longueur du terrain de BB) ou en colonnes d'attaquants (derrière la ligne de fond du terrain de BB) et de défenseurs (situés en attente sur la ligne de touche au milieu du terrain)] les empêchent, 


\section{eJRIEPS 24 juillet 2011}

la plupart du temps, de réaliser la tâche prescrite par MTV. Ce moment mène vers une impasse technique les élèves du MTV.

4. 2. 3. Le troisième moment du cycle: un retour sur les situations problématiques

Pendant la séance 8, MTV, voyant que l'échange n'apparaît pas dans la situation à effectif réduit en colonnes (en rose clair) décide de consacrer une séance à l'étude des objets Narier la distance de passe en fonction du rapport de force/ et /Renforcement de la passe dans le couloir de jeu central/ grâce à la situation à supériorité numérique (rose clair) et à la situation réduite de baby-foot (rose foncé). Les discussions qui suivent ces deux situations nous l'indiquent:

MTV : “ Où dois-je me placer si 3 passes ?"

é : «Passes en hauteur.»

MTV : «En moins de trois passes vous êtes là entrain de tenter !»

Cependant, si la construction de l'objet /Arriver vite en zone de marque/ est élaborée lors de la situation baby-foot (en rose foncé), la réalisation de la situation à supériorité numérique (en rose clair) ne va pas aller de soi et entraîne les élèves dans un échec. Nous imputons ce constat au fait que MTV ne modifie pas la configuration spatiale de cette situation qui facilite, pour MTV, la gestion du groupe classe (colonnes), mais il constitue une pierre d'achoppement pour les apprentissages des élèves.

4. 2. 4. Le quatrième moment : un travail consacré aux habiletés individuelles du basket-ball

Pendant les séances 9, MTV entre dans le travail des habiletés individuelles propres au basket, la passe, le tir et le dribble (colonne multi couleurs) dans des ateliers décontextualisés occupant toute la durée de la séance. Même si ce travail technique est repris pendant la séance 10, une seule séance lui est consacrée (S9) où les élèves dits forts doivent enseigner et aider les élèves dits faibles. Ce travail est en rupture avec les jeux (en rose et en vert) présents pendant toutes les séances du cycle.

4. 2. 5. Le cinquième moment : un bilan des objets travaillés pendant le cycle Pendant la séance 10, MTV reprend les deux objets /Varier la distance de passe en fonction du rapport de force/ et /Efficacité des habiletés passes, tirs et dribble/. II reprend les situations (en rose clair) qui ont été travaillées tout au long du cycle. Sa réalisation est très difficile ce qui amène MTV à prendre à sa charge un certain nombre de prescriptions :

MTV : «Edith tu n'as pas le droit de marcher avec la balle / ah tu te débrouilles un $\uparrow /$ Eloa dépêche toi $\uparrow /$ démarque toi Eloa $\uparrow / /$ allez là stop là allez/ démarque 


\section{eJRIEPS 24 juillet 2011}

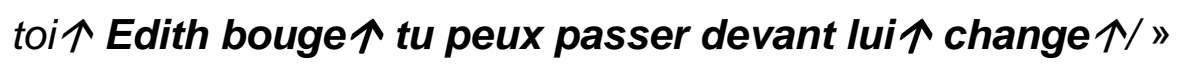

De même, comme nous l'avons analysé lors du deuxième moment de l'étude, les joueurs ont de grandes difficultés comme l'extrait, ci-dessous, l'indique :

MTV : “/ Non je ne sais pas moi/...c'est le pire que j'ai pu voir/// II y a une [seule] équipe qui est arrivée au panier cette fois ci "

4. 2. 6. Un sixième moment particulier : la relance du cycle

Face à ce constat et à la réflexion consécutive à l'entretien qui a suivi cette séance filmée, MTV prend conscience de la variable didactique équipe (variable forte chez MB). Son intention didactique est alors :

MTV : «Je leur demanderai d'aller vite, de jouer plus vite» (entretien post S10 vidéo)

MTV : «... Il y a aussi le fait qu'ils ne soient pas par équipe dans ce trois contre deux, ils ne construisent pas ensemble... » (entretien post S10 vidéo)

MTV ne modifie donc pas les équipes pendant la séance 11 qui est une séance d'apprentissage, contrairement aux autres sites, et constitue une rupture dans le cycle.

4. 2. 7. Conclusion sur les moments didactiques du MTV

Ce qui caractérise le cycle du MTV, ce sont des jeux (en rouge) ou des matches de BB (en vert) à toutes les séances du cyle sauf pendant la séance 9 qui concerne un travail technique sur /La maîtrise des habiletés techniques individuelles/ dans des ateliers décontextualisés occupant toute la durée de la séance. Par ailleurs, les situations qui illustrent le thème /Continuité du jeu en avant/ pendant le deuxième moment sont identiques à celles des troisième et quatrième moments. Soulignons aussi, à la lecture du tableau, que le travail de la technique, contrairement au MB, ne fait pas partie de la conception d'apprentissage du MTV. Ces moments didactiques autour desquels se structure le cycle n'obéissent pas vraiment à une logique d'évolution entre anciens et nouveaux savoirs (Chevallard, 1991).

4. 3. Analyse de la chronogenèse des objets enseignés du maître STAPS (MS) 


\section{eJRIEPS 24 juillet 2011}

Tableau III. Matrice de condensation des données de la chronogenèse des objets enseignés de MS

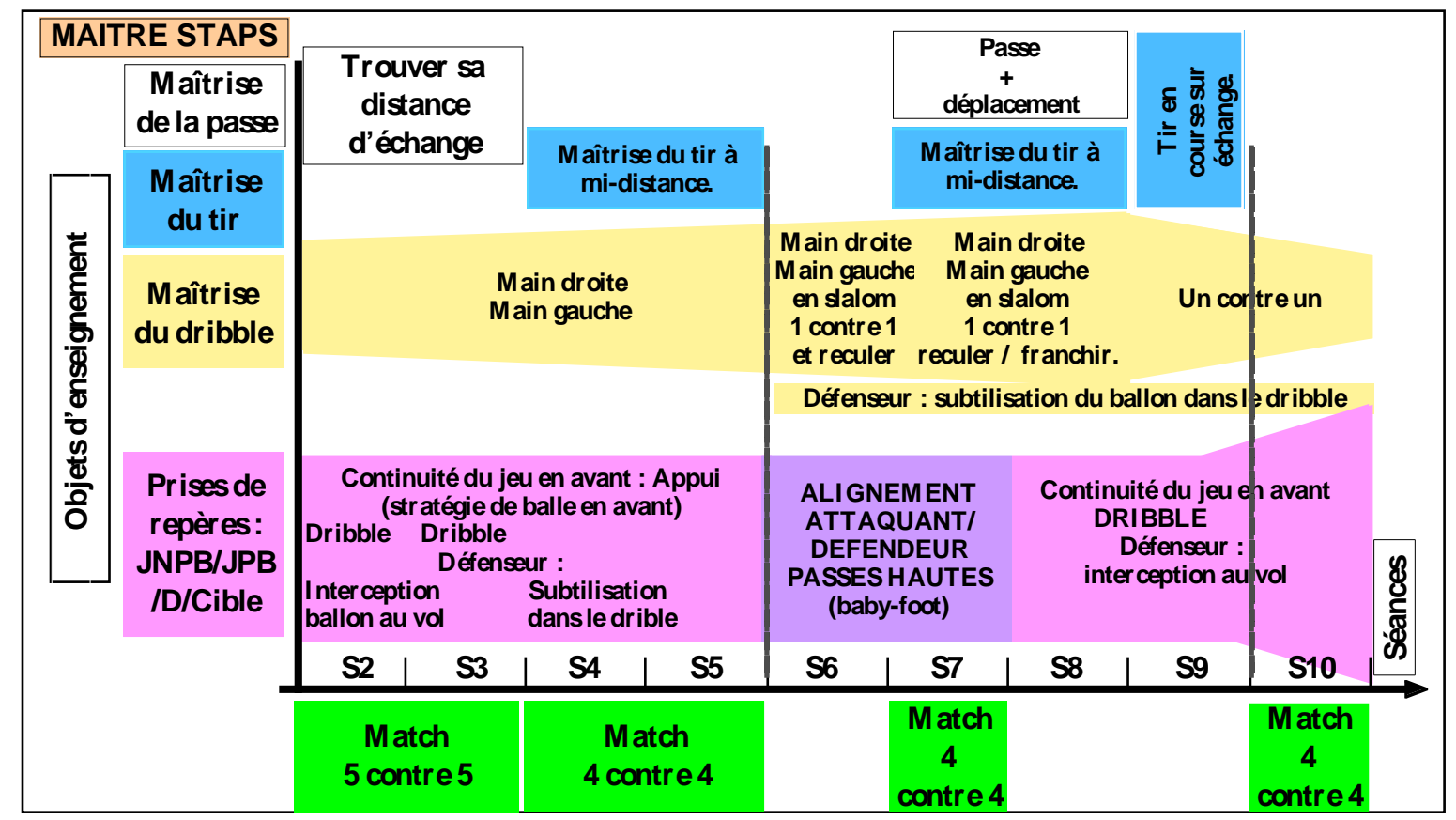

Comme MTV, MS met à l'étude différents objets de savoir tout au long du cycle. Notons cependant, comme le montre ce schéma, que la caractéristique essentielle du MS est la reprise systématique de deux d'entre eux tout au long du cycle : le dribble (en jaune) et la continuité du jeu en avant (en rose). Notre analyse indique une articulation majeure entre l'évaluation diagnostique et le thème /Les habiletés motrices individuelles/. L'observation effectuée pendant les matches amène «la classe » à évaluer le nombre d'habiletés motrices individuelles sur le nombre total des habiletés motrices réalisées par " la classe » pendant tous les matches de la séance. Les résultats de l'observation montrent de grandes difficultés dans le dribble et le tir, observation qui va jouer un rôle essentiel dans le projet du cycle du MS. II tient aussi compte du projet élaboré par les élèves pendant le bilan des matches, autour notamment de la réponse convenue représentée par le démarquage.

Quelle est l'articulation entre ces deux thèmes d'enseignement et les moments du cycle ? La condensation des données indique que si le dribble (en jaune) est enseigné pendant toutes les séances du cycle, il est interdit pendant l'étude du thème /Continuité du jeu en avant/. Par ailleurs, l'organisation des objets correspondant au thème /Conserver la balle pour aller tirer/ (en rose) comporte trois moments caractéristiques au cours du cycle du MS où nous avons observé un aller et retour entre les objets enseignés et les variables 


\section{eJRIEPS 24 juillet 2011}

didactiques, alors que les objets enseignés liés au thème /Les habiletés motrices individuelles/ sont découpés en quatre moments où ces objets sont envisagés selon une logique de complication (Marsenach, 1991) grâce une progression d'exercices.

4. 3. 1. Premier moment de l'étude : une première rencontre avec les thèmes d'enseignement du cycle

Nous interprétons que ce premier moment de l'étude comme une première rencontre avec les objets /Dribble/ (en jaune) et /Passe/ (en blanc) de la séance 2 à la séance 3, et l'objet /Continuité du jeu en avant avec une passe au joueur démarqué/ (en rose clair) mais de la séance 2 à la séance 5 .

II s'agit d'entrer dans les problèmes posés par la technique du dribble (en jaune) et de la distance d'échange (en blanc) dans des situations très décontextualisées (Chevallard, 1999) où l'observation montre que les élèves jouent l'adversité avec leur partenaire et nous interprétons ces « déviances » dans les ateliers proposés comme une redéfinition ludique des tâches, qui modifient le contrat didactique.

MS propose aussi, pendant ce moment de l'étude, une situation avec une supériorité numérique pour les attaquants (en rose clair). A l'instar du MTV, les modalités d'organisation géométrique de cette situation placent les joueurs en colonnes d'attaquants et de défenseurs. Cette organisation géométrique en colonnes ne permet pas aux joueurs de jouer en équipes. De plus, elle comporte une contrainte forte, l'interdiction de dribbler, ce qui est en contradiction avec le fait que le dribble est enseigné. Cette situation a donc de très faibles chances de permettre la construction de la contre attaque et les objets de savoirs constitutifs de la /Continuité de jeu en avant/ vont être difficilement rencontrés par les joueurs.

La permanence des équipes comme variable didactique essentielle de la construction d'un jeu collectif n'est pas prise en compte par MS contrairement au MB.

4. 3. 2. Deuxième moment de l'étude : maîtrise des habiletés individuelles

A partir de la séance 4, /La distance d'échange sur place/ (en blanc) est abandonnée au profit de l'objet /Tir/ (en bleu). L'objet /Dribble/ (en jaune) est conservé. Comme dans le moment précédent, pendant les séances 4 et 5, MS propose deux ateliers dans des tâches très décontextualisées qui permettent aux élèves de /Construire le point haut de trajectoire de tir en différents points de la raquette/ et de /Travailler le tempo du rebond du ballon dans la main, en fonction de l'allure du dribbleur/. MS fait alors élaborer, au cours des échanges dans les ateliers, les règles d'action par les élèves sur leurs actions en dribble et en tir. 


\section{eJRIEPS 24 juillet 2011}

Nous interprétons que MS construit, dans ce deuxième moment de l'étude uniquement le thème /Maîtrise des habiletés motrices individuelles/, une ébauche de travail technique de façon mécaniste de ces deux objets enseignés selon Brau-Antony (2001). Selon ce chercheur, dans ce type de conception, les habiletés motrices individuelles en BB sont enseignées pour elles-mêmes, indépendamment du jeu entre un attaquant et un défenseur.

4. 3. 3. Troisième moment de l'étude : une séance de départ commune aux deux thèmes

Nous en avons situé un moment critique commun aux deux thèmes d'étude (/Conserver la balle pour aller tirer/ en rose et /Maîtrise du dribble/ en jaune) à la sixième séance du cycle. Cette rupture correspond à un travail technique pour les deux thèmes d'enseignement. MS entre en effet, à partir de la séance 6 , dans un travail où il va /Perfectionner les habiletés motrices individuelles/ et plus particulièrement l'objet /Dribble/ avec une logique de complication comme le montre le tableau (en jaune de S6 à S8) cidessus. Par exemple, un atelier passe se complique: il s'agit de /Passer puis de se déplacer pour s'écarter du nouveau joueur porteur de balle/ Nous retrouvons l'idée /d'Ecarter l'espace de jeu dans le sens latéral/ que MS souhaite voir apparaître lors de la situation didactique du deuxième thème continuité du jeu en avant comme il nous l'indique dans l'entretien post de la séance 7 :

MS : "Le passe et va, c'était oui de toute façon dans la situation est quand même assez fermée euh mais déjà y'avait une action intellectuelle quand même à faire, je passe à droite mais j'y vais pas, je vais à gauche donc... ça désamorce des automatismes et ça ouvre d'autres possibilités et après sur le jeu donc c'était se placer pour offrir des possibilités à celui qui a le ballon... » (MS, entretien post, S7)

Cet extrait d'entretien indique que MS est tout à fait conscient des rapports entre acquisition d'une habileté motrice et développement des alternatives tactiques, sans pour cela les mettre explicitement à l'étude. En effet, de la séance 6 à la séance 7, MS met à l'étude la /Construction de la trajectoire de passe haute au-dessus du défenseur/ (en rose). Dans ce dessein, il propose, à l'instar du MTV, un dispositif qui place les joueurs dans une configuration géométrique (le baby-foot en rose foncé) qui n'est pas connexe avec «le passe et va » et la tâche présentée (le baby-foot) va devenir quasi routinière (Chevallard, 1999) car le défenseur n'est pas très actif. 
eJRIEPS 24 juillet 2011

4. 3. 4. Quatrième moment de la séance 9 à la séance $10:$ une dissociation des thèmes d'enseignement

Pendant la séance 9 , MS recontextualise le travail de la technique du dribble. L'objet /Enchaîner les habiletés individuelles en vue d'un but/ est mis à l'étude dans les ateliers tir (en bleu) et dribble (en jaune). Par exemple, l'objet /Utiliser la variation de l'allure de la course du dribbleur pour créer un dispositif attaquant défenseur pendant $20 \mathrm{~m} /$ est proposé. Cet atelier de dribble demande une forte technicité qui est ici renforcée avec la présence du défenseur. Nous avons d'ailleurs observé que les attaquants perdent souvent la balle. Cette recontextualisation des objets correspond, d'après nous, à un bilan des savoirs relatifs aux /Habiletés individuelles/ et plus particulièrement à l'objet emblématique du cycle du MS le /Dribble/.

Mais, c'est de la séance 8 à la séance 10 que MS reprend la situation didactique 3vs2 (en rose clair) déjà travaillée lors du premier moment de l'étude, et il réintroduit de nouveau une contrainte didactique forte, l'interdiction de dribbler. Nous avons d'ailleurs observé que, pendant la séance 10, toutes les situations proposées sont contextualisées ce qui correspond à une rupture (comme l'indique le tableau : trait noir) par rapport aux autres séances du cycle qui contiennent des exercices décontextualisés. Nonobstant, le blocage que nous avons signalé dans le premier moment du cycle pendant la situation 3 vs 2 se reproduit (les deux défenseurs se déplacent sur les partenaires du joueur porteur de balle). Nous considérons du fait des contraintes du milieu choisi que la situation empêche les élèves de développer une stratégie de balle en avant. Ces quelques points signalent, selon nous, une difficulté du MS, déjà évoquée à propos des habiletés motrices, qui ne semble pas pouvoir anticiper les conséquences des dispositifs proposés.

\section{3. 5. Conclusion sur la chronogenèse du maître STAPS}

Nous avons mis en évidence que les deux objets emblématiques du cycle (le dribble en jaune et le jeu en rose) n'étaient pas clairement articulés dans le processus d'enseignement et sont l'objet de ruptures didactiques. Le travail plus systématique de la technique qui concerne la passe (en blanc) et le tir (en bleu) permet cependant à MS de pouvoir articuler l'ensemble très formellement et partiellement en fin de cycle.

4. 4. Analyse de la chronogenèse des objets enseignés du maître et de l'intervenant extérieur (M/IE)

Comme le synthétise le tableau ci-dessous, l'organisation temporelle du M/IE est caractérisée par l'extrême éclatement des objets de savoir dans le cycle dont l'intervenant 


\section{eJRIEPS 24 juillet 2011}

extérieur (IE) est à l'origine. A contrario du MB, sept objets d'enseignement sont successivement mis l'étude et ils sont entrecoupés de séances de matches (en vert).

Tableau IV. Matrice de condensation des données : chronogenèse des objets enseignés de l'intervenant extérieur.

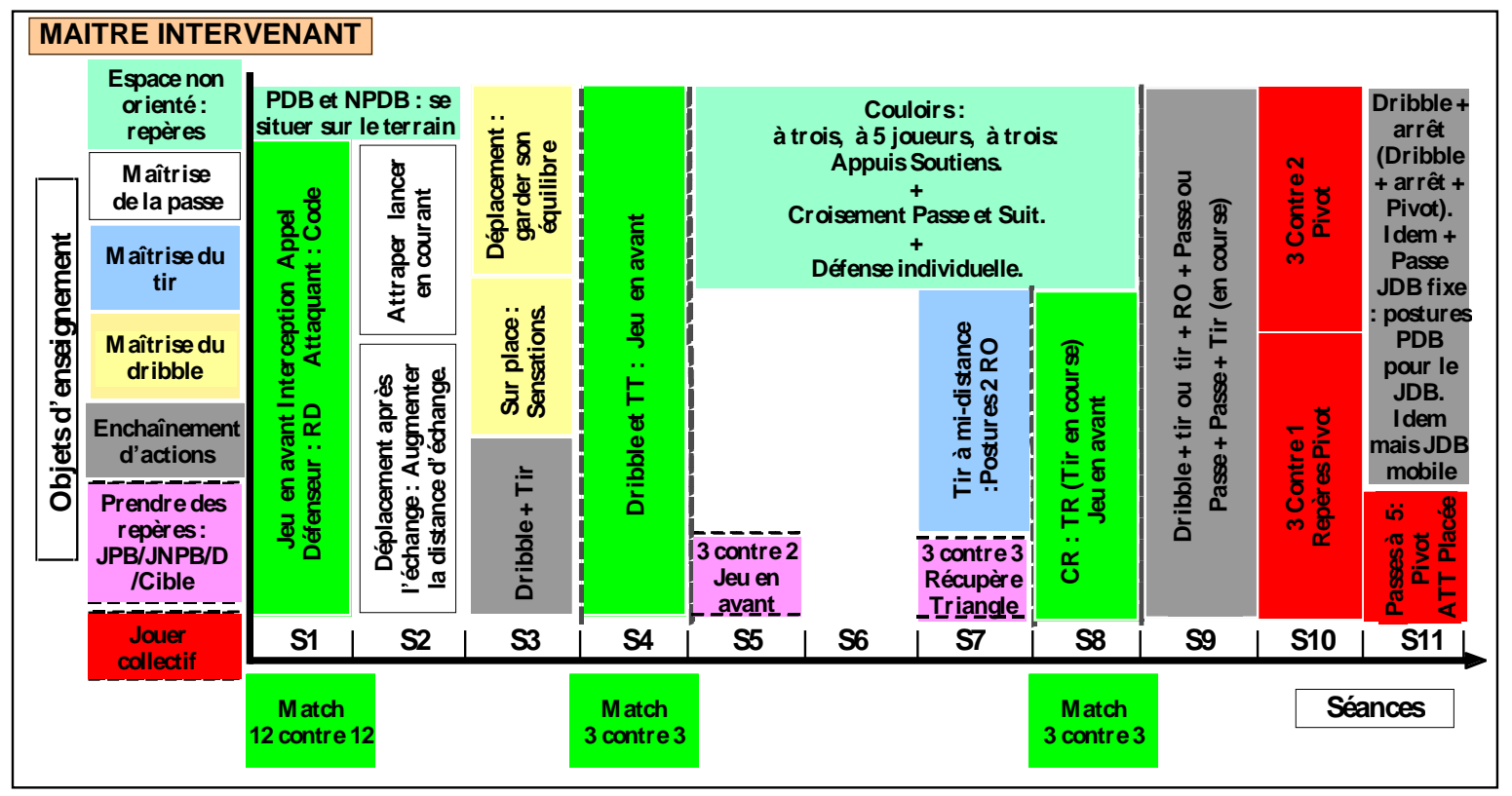

La condensation des données met en évidence cinq thèmes d'enseignement différents pendant le cycle dont trois sont plus particulièrement déclinés pendant le cycle. Notons, tout de suite, que le thème négocié /Conserver la balle pour aller tirer en élargissant l'espace de jeu/ (en rose), qui appartient au contrat de recherche, est mis à l'étude de manière épisodique pendant le cycle grâce à une situation avec supériorité numérique pour l'équipe attaquante en séance 5 et à une situation à effectif réduit 3 vs 3 en séance 7. Nous avons observé que seuls quelques élèves peuvent s'impliquer dans cette situation (rose pointillé). A contrario, trois thèmes sont clairement abordés pendant le cycle par IE : le thème /Habiletés motrices propres au BB/ (la passe en blanc, le dribble en jaune et le tir en bleu), le thème /Enchaînement des habiletés motrices/ (en gris), et celui /Prendre des repères dans un espace de jeu non orienté/ (en turquoise). Nous avons observé que ces trois thèmes d'enseignement font partie du projet d'enseignement d'IE :

IE : « Le sport co je le vois comme ça, le ballon dans les mains, il faut toucher un maximum de ballon, il faut être à l'aise avec le ballon. Toute la manipulation du ballon, quand on joue au basket donc il est important de savoir le manipuler. » (IE entretien protocole) 


\section{eJRIEPS 24 juillet 2011}

En contrepoint de ces trois thèmes d'enseignement, se superpose un thème qui concerne le /Jouer collectivement/ (en rouge).

Nous avons observé qu'aucun de ces thèmes d'enseignement n'était en continuité pendant tout le cycle. Ils sont mis à l'étude séance par séance selon une logique transpositive cumulative (Marsenach, 1991), et nous avons d'ailleurs observé une rupture didactique entre toutes les séances du cycle d'IE sauf pendant les séances 5 et 6 où nous avons observé que le thème /Prendre des repères dans l'espace de jeu non orienté/ (en turquoise) est un thème important à travailler pour IE. Ses interactions avec les élèves vont dans ce sens et sont opérantes dans les matches (en vert) qui pour IE sont le moment de la mise en application des « fondamentaux techniques » :

IE : "J'envisage de les faire jouer: on a vu les fondamentaux: la passe, le dribble, un petit peu de tir. A partir de là, on va les lâcher sur le terrain et on va leur dire: maintenant avec ce que vous savez, essayez de jouer. " (S3 vidéo entretien post)

IE introduit en effet de manière régulière dans son cycle un match (en vert) de trois attaquants contre trois défenseurs (S4, S8 et S12), objet de la seconde rupture du cycle. Nous commençons par décrire chacun des trois thèmes explicites pour IE. Puis nous tenterons lors de la conclusion de les articuler avec les deux autres thèmes qui sont apparus plus implicitement chez cet enseignant (élargir l'espace de jeu et jouer collectivement).

4. 4. 1. Organisation des trois thèmes explicites d'IE dans le cycle Le thème sur les habiletés motrices propres au $B B$ L'objet /Passe/ (en blanc), puis l'objet /Dribble/ (en jaune) et ensuite l'objet /Tir à midistance/ (en bleu) sont mis à l'étude successivement à l'étude pendant trois séances. II structure les moments de l'étude des trois objets (l'introduction, le développement et la conclusion) sur une seule séance sans tenir compte des incidents qui émaillent l'évolution du dispositif envisagé a priori. Les exercices proposés au cours des trois séances sont en dehors du contexte du jeu c'est-à-dire sans une mise à l'étude en situation de rapport de force avec des défenseurs. L'évaluation de l'objet enseigné au cours la séance consiste en un jeu décontextualisé (Chevallard, 1991). Cette façon de concevoir soutient une conception très mécaniste (Mérand, 1989) qui sous-tend l'action d'IE. Par ailleurs, nous avons observé que cet objet /Tir/ (en bleu) était enseigné cinq séances après les objets /Passe/ et /Dribble/ et était notamment articulé avec celui de l'articulation des deux autres. 


\section{eJRIEPS 24 juillet 2011}

Le thème enchaînement des habiletés individuelles

Ce thème d'enseignement (en gris dans le tableau) apparaît dans le cycle d'lE dès la troisième séance. L'analyse de ses objets enseignés montre, par croisement avec celles explicites des habiletés motrices, la logique de construction des objets enseignés suivie par IE. II débute tout d'abord le cycle par l'étude de chaque habileté [S2 (la passe), S3 (le dribble) puis S7 (le tir), ensuite, à partir de la séance 3 (S3, S7 et S9)], il entre dans un travail d'association de ces habiletés qu'il conclut pendant la séance 9 (toute en gris).

IE : «II faut d'abord supprimer l'adversaire sinon cela fait trop de chose à la fois. La passe, le déplacement déjà, tu imagines ? Déjà, se déplacer faire monter un ballon, l'amener d'un panier à un autre, sans avoir à penser à cette gêne qu'est la défense. " (IE entretien protocole)

Enfin il relance ces enchaînements (S11, le pivot) dans un contexte présentant une incertitude qui permet un nouveau travail centré à nouveau sur le JPB.

IE : «Dans un jeu, sans arrêt, on a le ballon dans les mains, il est important ce ballon, on le maîtrise sous toutes ses formes dans toutes les positions. ” (IE, entretien protocole)

Cette accélération du temps didactique chez IE, indépendamment du temps d'apprentissage des élèves est significative d'une conception cumulative des apprentissages scolaires. Ainsi sommes-nous dans « une fiction d'un temps didactique unique » (Chevallard, 1991). Par ailleurs, toutes les situations proposées pour ces enchaînements d'action sont décontextualisés, ce qui confirme encore, selon nous, une «conception mécaniste et associationniste de l'apprentissage » telle que mise en évidence dans le film de Barrais (“à l'école du basket » (1963) critiqué par les élèves de $3^{\text {ème }}$ année de l'ENSEP). L'intelligibilité de cet éparpillement nous est donnée par l'étude du thème d'enseignement ci-après.

Les objets enseignés liés au thème /Prendre des repères dans un espace de jeu non orientél.

Le thème d'enseignement, /Prendre des repères dans un espace de jeu non orienté/ (bleu turquoise) est étudié pendant les séances 5,6 et 8 . II concerne la prise de repères topographiques par les joueurs sur le terrain qui est orienté ou non orienté. Le porteur de balle est pris comme point fixe dans le couloir central de jeu, ses partenaires doivent se placer dans des couloirs parallèles : tout d'abord en triangle puis en carré autour du PDB. Comme les élèves se dissipent, il recontextualise pour montrer comment le triangle est 


\section{eJRIEPS 24 juillet 2011}

utilisable en situation de supériorité numérique 3 contre 2 (rose pointillé) lors de la séance 5. II s'agit d'une démonstration où l'équipe attaquante joue balle en avant et non en triangle. IE est donc piégé par le jeu des élèves au même titre que les joueurs subissent une rupture de contrat didactique entre l'objet de la séance et la situation ludique de fin de séance car son rapport aux savoirs ne lui permet pas d'avoir la main dessus. En effet, les trois attaquants jouent un gagne-terrain (/ continuité du jeu avant/) en dépassant les deux défenseurs et non un placement en carré avec défenseurs passifs (/prise de repères sur le terrain autour du porteur de balle/) comme ils ont appris pendant les séances (rupture entre les objets enseignés au cours de la séance).

\section{4. 2. Conclusion sur les thèmes d'enseignement du cycle}

La mise en scène temporelle des différents objets de savoir que nous avons mis en évidence va prendre ici tout son sens du point de vue d'IE. En effet, l'analyse de leur enchaînement entre les différentes séances nous donne la clef de sa logique de construction du cycle. Celui-ci démarre par l'enseignement des /Deux habiletés individuelles/, la passe (en blanc, S2) et le dribble (en jaune, S3), puis celles-ci vont être utiles pour /Se déplacer dans l'espace de jeu/ (S5 et S6, en turquoise). IE juge alors que pour être efficace dans cet espace, «il faut apprendre à marquer un panier » (S7, en bleu). Ensuite, pour arriver en zone de marque il faut /Enchaîner les habiletés individuelles/ apprises (S9, en gris). Enfin le JPB doit /Voir où sont ses partenaires dans l'espace de jeu/ (S11, en gris, le pivot) qui a été structuré pendant les séances 5 et 6 (en turquoise). Nous avons donc un enchaînement remarquable des différents objets enseignés au fil du cycle, compatible la mise en place d'une progression d'objectifs comme « suite ininterrompue graduelle » (Marsenach, 1991, p.24).

L'entretien protocole nous révèle l'origine de cette structuration, séance après séance où chacune d'entre elles est consacrée à un seul objet :

“On reste plus longtemps sur un exercice en club. Plus d'une séance, parce qu'il y a le match après, donc on en fait un peu plus. En club tu as toujours le match, la compétition. 》 (Entretien protocole IE)

Nous pensons qu'IE transpose à l'école, pendant un temps très court, les usages fédéraux tels qu'il les met en œuvre en club et qui s'organise sur le temps très long de la formation des joueurs (plusieurs années).

\section{Discussion. Comparaison des quatre cycles, points communs et différences selon leurs rapports aux savoirs}




\section{eJRIEPS 24 juillet 2011}

Les cycles du MB et du MTV sont découpés en différents moments qui articulent les objets d'enseignement du cycle, même si l'organisation didactique du MTV consiste en une reprise de ces objets et des situations qui les illustrent. Par contre, les deux thèmes d'étude du cycle du MS suivent une structuration didactique différente : l'analyse des différents moments du thème /Habiletés individuelles/ est particulièrement claire et est menée parallèlement au thème /Conserver la balle pour aller tirer en écartant l'espace de jeu/. L'analyse du cycle de l'IE se démarque de celle des trois maîtres. Elle met en évidence une logique d'étude isolée d'un objet d'enseignement dans chacune des séances, et leur compilation dans le cycle, car les objets mis à l'étude sont beaucoup plus nombreux que dans les autres sites (surtout par rapport aux MB et MS).

Le thème d'étude /Habiletés motrices individuelles/, et plus particulièrement l'objet /Dribble/ en continuité sur le cycle du MS suivent une logique identique à celle du travail du dribble chez IE et indique, selon nous, un rapport « mécaniste » au basket-ball du MS et d'IE. Cependant MS se détache d'IE car parallèlement au travail des habiletés individuelles, ce maître aborde, tout au long du cycle, une situation à supériorité numérique, et une situation réduite. Ces situations ont pour fonction de recontextualiser les objets de savoir étudiés. Par contre, nous pensons que la configuration topographique en colonnes d'attaquants et l'interdiction de dribbler révèlent la conception mécaniste qui sous-tend son travail, ce qui se traduit par une transformation de la situation en situation de jeu sur-réglé (Mérand, 1989; Brau-Antony, 2001). La conception topographique de la tactique du MS (à droite, puis à gauche dans le passe et va) renforce cette interprétation sur le mécanisme. N'oublions pas toutefois que d'un point de vue intrinsèque, ces situations envisagées comme un « jeu à thème » ont pour but de faire émerger les règles d'action dans une situation extrêmement déconnectée du rapport de force (le baby-foot) :

MS: «J'espérais que là, que la situation se bloquerait parce que s'ils [les attaquants] s'arrêtaient suffisamment tôt face au défenseur, le défenseur ne peut pas les attaquer, si les autres [JNPB] se placent bien entre les deux zones la passe va se faire et si le défenseur se déplace à gauche il [JPB] passe à droite... Ils avaient bien compris que, ce qu'on avait travaillé avant devait fonctionner, devenait intéressant, ils commençaient à I'intellectualiser... » (MS, S7 vidéo, entretien post)devenait intéressant, ils commençaient à l'intellectualiser... » (MS, S7 vidéo, entretien post)

Ce point de vue intrinsèque témoigne en revanche d'une conception structuraliste telle que Brau Anthony (2001) la définit. 


\section{eJRIEPS 24 juillet 2011}

Le rapport aux savoirs du basket-ball oscille ainsi chez MS, suivant le contexte, entre une conception «mécaniste et une conception «structurale " (Brau Anthony, 2001), ce qui le différentie d'IE. Nous considérons que la conception structurale du MS témoigne de son assujettissement à la formation en STAPS qui, de notre point de vue, est en concurrence avec les contenus d'enseignement qu'il propose selon une conception mécaniste.

Contrairement au MS, la structure du cycle du MTV articule les objets d'enseignement du cycle. Ce maître commence son cycle par des jeux collectifs qui révèlent son attachement à l'institution du premier degré. Apparaît ici le conflit entre le rapport institutionnel à la formation didactique de l'IUFM qui l'invite à faire progresser les élèves, point commun avec $\mathrm{MB}$, et le rapport personnel à la conception éducative (le bien être des élèves à vivre ensemble) :

MTV : «Je pense que pour que les enfants progressent, il faut qu'ils prennent du plaisir et s'il n'y a pas de jeu et si à un moment donné on ne passe par le jeu au bout d'une séance, ils n'ont pas l'impression d'avoir bien participé, d'avoir fait ce sport qu'ils aiment en fait puisqu'ils aiment jouer ensemble. Cela me semble important " (MTV, entretien protocole).

II présente aussi des similarités avec MS : les situations illustrant les objets /Varier la distance de passe en fonction du rapport de force/ placent les élèves dans une conception topographique semblable (colonnes d'attaquants et de défenseurs). II s'en détache cependant car contrairement au MS, MTV a une proximité avec MB car il n'oublie pas le gain du jeu et cherche à faire progresser les élèves en hésitant, en proposant un allerretour entre la situation de jeu (en rose clair) et la situation avec blocage des défenseurs sur une ligne (en rose foncé), tout en valorisant le «jouer collectif ». Le travail de la technique chez MTV ne dure qu'une séance et arrive tardivement dans le cycle contrairement au MB (quatre séances) et au MS (trois séances) et il concerne les /Habiletés motrices individuelles/ à l'instar du MS. Nous suggérons que l'expérience sportive des MB et MS n'est pas sans lien avec ce choix du travail de la technique. Celuici porte chez MB, rappelons-le, sur les enjeux de savoir collectifs, ce qui n'est pas le cas chez MS. Les deux thèmes d'étude développés tout au long du cycle du MB sont articulés comme pour MTV. Contrairement à ce dernier, MB fait avancer le temps didactique. Cette façon d'articuler les objets d'enseignement dans le cycle est envisagée dans des situations à supériorité numérique où chaque joueur doit envisager des prises de décisions, conditionnées par un but commun : avoir un rapport au critère de réussite supérieur à l'équipe adverse : 
eJRIEPS 24 juillet 2011

$M B$ : « Je crois que là ils ont une marge de progression sur cet exercice [2 contre 1], sur la stratégie: Partir vite, mais si on s'aperçoit que si le défenseur part vite lui aussi ça veut dire être capable de s'arrêter à un moment donné. S'arrêter, une fois, deux fois ça va marcher, le défenseur là il fait trois pas de plus parce qu'il est dans son action, donc je m'arrête : ça veut dire quoi ? soit tenter un shoot à mi-distance, soi passer en un contre un : ça amène deux situations possibles, c'est à eux de voir » (MB, S6 entretien post).

L'organisation didactique du MB et cet extrait didactique sont révélateurs d'un rapport au basket inspiré d'une « conception dialectique » (Mérand, 1989, Vandevelde, 2001 et BrauAntony, 2001).

\section{Conclusion : quelques pistes pour la formation}

Notre recherche a mis en évidence la relation entre la variabilité des contenus d'enseignement et les multiples rapports aux savoirs des enseignants. L'activité professorale comprend deux dimensions qu'il s'agit de prendre en considération. II s'agit tout d'abord de la conception des dispositifs d'aide à l'étude qui permet aux professeurs d'école de saisir la spécificité des savoirs en contextes. Travailler avec les professeurs d'école sur l'agencement des milieux didactiques nous semble nécessaire et relève des usages de formation très répandus dans les IUFM. Mais le travail de conception de dispositifs ouverts doit aussi envisager les potentielles actions motrices des élèves en situation, d'où la nécessité d'armer le regard des professeurs stagiaires en terme d'analyse a priori des situations qu'ils conçoivent. Cette seconde dimension nous semble insuffisamment développée dans la formation EPS du premier degré. Enfin, l'apport de notre travail consiste, après d'autres, à considérer que la direction d'étude en contexte est fondamentale pour «armer »le regard des professeurs d'école en EPS (Vandevelde, 2001, Amade-Escot, 1997 et Marsenach et Mérand, 1987).

\section{Bibliographie}

Amade-Escot \& C., Marsenach, J. (1995). Didactique de l'éducation physique et sportive. Paris : La pensée sauvage.

Amade-Escot \& C., Leziart, Y. (1996). Contribution à l'étude de la diffusion de propositions d'ingénierie didactique auprès de praticien. Analyse de cas d'enseignants d'éducation physique et sportive volontaires. Recherche INRP N30506, unité EPS du département « didactiques des disciplines ». 
eJRIEPS 24 juillet 2011

Amade-Escot, C. (1997). Observation des situations didactiques et pratique réflexive en formation initiale. Recherche et formation, 25, 47-56.

Amade-Escot, C. (1998). L'enseignant d'EPS dans les interactions didactiques. Itinéraire de recherche. Habilitation à diriger des recherches non publiée. Toulouse : Université de Toulouse 3.

Amade-Escot, C. \& Sensevy, G. (2002). Relations chercheurs-praticiens dans l'observation des pratiques enseignantes. Document interne (pp. 1-5). Réseau OPEN de l'ACl : «Education et formation » dirigé par M. Altet, M. Bru, et C. Blanchard-Laville.

Amade-Escot C., Amans-Passaga, C. \& Montaud D. (2009). Les savoirs mobilisés dans l'action didactique par les intervenants en activités physiques et sportives : un entrelacs de processus épistémiques et institutionnels. Sciences de la Société, 77, 43-62.

Baillat, G.,\& Guillon, R. (1998). Polyvalence et EPS dans l'enseignement du premier degré. Contre Pied, 3, 6-12.

Brau-Antony, S. (2001). Les conceptions des enseignants d'éducation physique et sportive sur l'enseignement et l'évaluation des jeux sportifs collectifs : résultats d'une enquête. STAPS, 56, 93-108.

Briere-Guenoun, F. (2005). De l'observation des pratiques aux connaissances mobilisées par le professeur dans l'interaction didactique: Le cas du franchissement par redressement au saut de cheval. Thèse de doctorat en STAPS, non publiée. Orléans: Université d'Orléans.

Chevallard, Y. (1989). Le concept de rapport au savoir, rapport personnel, rapport institutionnel, rapport officiel. Document interne (pp 211-235). IREM d'Aix Marseille: Faculté des sciences de Luminy.

Chevallard, Y. (1991). Postface à la deuxième édition, didactique, anthropologie, mathématiques. In Y. Chevallard, La transposition didactique, du savoir savant au savoir enseigné (pp. 199-234). Grenoble : Edition la pensée sauvage.

Chevallard, Y. (1992). Concepts fondamentaux de la didactique : perspectives apportées par une approche anthropologique. Recherches en Didactiques des Mathématiques, Vol 12,1, 73-112.

Chevallard, Y. (1999). L'analyse des pratiques enseignantes en Théorie Anthropologie Didactique. Recherches en Didactiques des Mathématiques, Vol 19, 2, 221-266.

Durand, M. (1998). Efficacité et expertise en enseignement : réflexion méthodologique et théorique. In C. Amade-Escot, J-P. Barrué \& A. Terrisse (Eds.), Recherche en EPS. Bilans et perspectives (pp. 39-56). Paris : Editions Revue EPS. 


\section{eJRIEPS 24 juillet 2011}

Elèves de $3^{\text {ème }}$ année ENSEP. (1963). Etude critique du film «à l'école du basket-ball ». Document interne ronéoté ENSEP.

Huberman, M. \& Miles, M. (1991). Analyses des données qualitatives. Bruxelles : De Boëck.

Leutenegger, F. (2000). L'observation en classe ordinaire de mathématiques. In Résumés des Actes du colloque international, Autour de la Théorie des Situations Didactiques, livret de contributions (pp. 122-129). Bordeaux, Université Bordeaux V. Ségalen.

Marsenach, J., Mérand, R. (1987). L'évaluation formative en EPS dans les collèges. Rapport scientifique. N2. Paris : INRP.

Marsenach, J. (1991). L'EPS Quel enseignement ? Paris : INRP.

Mérand, R. (1989). La rénovation des contenus d'enseignement : jeux sportifs au collège. Revue Française de Pédagogie, 89, 11-14.

Schubauer-Leoni, M.L. \& Leutenegger, F. (2002). Expliquer et comprendre dans une approche clinique/expérimentale du didactique ordinaire. In F. Leutenegger \& M. SaadaRobert (Eds.), Expliquer et comprendre en sciences de l'éducation (pp. 227-251). Bruxelles: De Boëck, Raisons éducatives.

Schubauer-Leoni, M.L. (2006). La construction de la référence dans l'action conjointe professeur-élève. In N. Wallian, M.P. Poggi \& M. Musard (Eds.), Co-construire des savoirs (pp. 67-85). Besançon : Presse Universitaire de Franche-Comté.

Sensevy, G. (2007). Des catégories pour décrire et comprendre l'action didactique. In G, Sensevy \& A. Mercier (Eds.), Agir ensemble. L'action conjointe en didactique (pp. 67-85). Rennes : Presse Universitaire de Rennes.

Vandevelde, M. (2001). L'action de formation d'Alès en basket-ball: contribution à l'analyse du système didactique de formation continue en EPS. Thèse de doctorat non publiée, Sciences de l'éducation. Toulouse : Université de Toulouse III. 